\title{
THE FUNDAMENTAL GROUP OF NON-NEGATIVELY CURVED MANIFOLDS
}

\author{
David Wraith
}

The aim of this article is to offer a brief survey of an interesting, yet accessible line of research in Differential Geometry. A fundamental problem of mathematics is to understand the relationship between the geometry and topology of manifolds. The geometry of a manifold is determined by a Riemannian metric-that is, a smoothly varying inner product on the tangent bundle. Altering the Riemannian metric on a given manifold alters the way in which it curves. It is natural, therefore, to ask to what extent the possible curvatures of a manifold determine and are determined by its topology. Note that all manifolds are assumed to be Riemannian, smooth, complete and without boundary.

There are three main ways of quantifying curvature: sectional curvature, Ricci curvature and scalar curvature. The sectional curvature provides the most information, and the scalar curvature the least. The Ricci curvature can be thought of as an average of sectional curvatures, and the scalar curvature in turn as an average of Ricci curvatures. Any textbook on Differential Geometry can be consulted for the exact definitions.

Of particular importance is the sign of the curvature. For example, in dimensions $\geq 3$ every manifold admits a metric of negative Ricci (and therefore negative scalar) curvature. On the other hand, relatively few possess metrics of everywhere positive curvature. Manifolds that admit for example a positive Ricci curvature metric include spheres, some algebraic varieties, many exotic spheres, Lie groups and homogeneous spaces. Much is now known about how the existence of a positive scalar curvature metric restricts the possible topology of the manifold. Comparatively 
little is known about positive or non-negative Ricci and sectional curvature. (See [7] for an overall view.) For an introduction to the relevance of curvature conditions for the local and global geometry, see $[9]$.

The object of our attention will be the influence of positive or non-negative curvature on perhaps the simplest topological invariant: the fundamental group. There are many results describing this influence, the most important of which we will touch on below.

There are two approaches to this line of study. One approach is to attempt to describe the structure of $\pi_{1}$, and in particular to say something about the kind of subgroups which can arise. The other approach is to investigate the growth of $\pi_{1}$, a concept which we will explain presently.

Consider a finitely generated group. We will define the growth of such a group. Let $\left\{g_{1}, g_{2}, \ldots, g_{r}\right\}$ be a set of generators. Let $\gamma(s)$, $n \in \mathbb{N}$ be the number of group elements which can be expressed as words of length $\leq s$ in the generators and their inverses. Clearly, $\gamma(s)$ will depend on the particular choice of generators. However, the way in which $\gamma(s)$ behaves as $s \longrightarrow \infty$ turns out to be essentially independent of this choice

We will say that the growth of the group is exponential if $\gamma(s) \geq a^{s}$ for some constant $a>1$. We will say the growth is polynomial if $\gamma(s) \leq c s^{d}$ for some constants $c$ and $d$. More precisely we will say that the group has polynomial growth of order $\leq p$ if

$$
\limsup _{s \rightarrow \infty} \frac{\gamma(s)}{s^{p}}<\infty
$$

and has polynomial growth of order $\geq p$ if

$$
\liminf _{s \rightarrow \infty} \frac{\gamma(s)}{s^{p}}>0
$$

It is straightforward to show that $\mathbb{Z}^{n}$ has polynomial growth of order $n$. (For example, $\mathbb{Z} \oplus \mathbb{Z}$ has growth function $\gamma(s)=2 s^{2}+$ $2 s+1$.) In contrast, the free group on $r$ generators has exponential 
growth. Explicitly $G p\left\{g_{1}, g_{2}, \ldots, g_{r}\right\}$ has growth function

$$
\gamma(s)=1+\frac{r}{r-1}\left[(2 r-1)^{s}-1\right] .
$$

It can be shown that these concepts are independent of the chosen generators.

To help understand the implications of group growth, it is useful to consider the growth of a finitely generated abelian group with generators $g_{1}, \ldots, g_{r}$. It is clear that the growth function $\gamma(s)$ is less than or equal to the corresponding function for the free abelian group on $r$ generators, which by the above has polynomial growth of order $r$. It follows then that a finitely generated abelian group has polynomial growth of order at most equal to the minimum possible number of generators. From this we can also conclude that a finitely generated group with exponential growth must be non-abelian.

We begin our survey by considering the weakest measure of curvature: the scalar curvature.

As remarked earlier, the property of admitting a positive or non-negative scalar curvature metric imposes restrictions on the possible topology of the underlying manifold. This is particularly true in the compact case (see [15] for example). However, the influence of the scalar curvature on the fundamental group is somewhat weaker than might be expected.

Let $(M, g)$ be any Riemannian manifold, compact or noncompact. (In the non-compact case assume the scalar curvature is bounded above and below.) Then there is a metric $g_{1}$ on $M \times S^{2}$ such that $\operatorname{scal}\left(g_{1}\right)>0$. The point here is that $\pi_{1}(M) \cong \pi_{1}(M \times$ $S^{2}$ ), so the fundamental group alone cannot be an obstruction to positive scalar curvature. We define $g_{1}$ to be a product metric as follows:

$$
g_{1}=g+t d s^{2}
$$

where $d s^{2}$ is the round metric of radius 1 on $S^{2}$ and $t$ is a constant. We have

$$
\operatorname{scal}\left(g_{1}\right)=\operatorname{scal}(g)+\frac{2}{t} .
$$


We can therefore ensure $\operatorname{scal}\left(g_{1}\right)>0$ everywhere simply by choosing $t$ sufficiently small.

Given that scalar curvature restrictions hold little interest for our considerations, it makes sense to consider instead the Ricci curvature.

The most famous result in this direction is due to Myers: a compact manifold with positive Ricci curvature has a finite fundamental group.

If we weaken the condition Ric $>0$ to Ric $\geq 0$, then Myers' result no longer holds. For example if $M$ has a positive Ricci curvature metric, the product metric on $M \times S^{1}$ has non- negative Ricci curvature (where $S^{1}$ is equipped with the standard metric). However, $\pi_{1}\left(M \times S^{1}\right) \cong \pi_{1}(M) \oplus \mathbb{Z}$. Of course the class Ric $\geq 0$ also contains flat tori, and $\pi_{1}\left(T^{n}\right) \cong \mathbb{Z}^{n}$. More generally, it can be shown that the fundamental group of a compact $n$-dimensional manifold $M$ has a free abelian subgroup of rank $\leq n$ of finite index in $\pi_{1}(M)$. This is a consequence of the Splitting Theorem [6], which states that if $M$ is a non-compact, Ricci non-negative manifold which contains a line (ie a non-closed geodesic) then $M$ is isometric to some metric product $M^{\prime} \times \mathbb{R}$. In terms of group growth, we can deduce that $\pi_{1}(M)$ has polynomial growth of order $\leq n$.

For Ricci positive or non-negative curvature, the non-compact case is more interesting. We begin by looking at Ric $\geq 0$.

The fundamental group of a non-compact manifold could be infinitely generated. Of course, the concept of group growth has no meaning in this case. However, if the Ricci curvature of an $n$-dimensional non-compact manifold $M$ is $\geq 0$, any finitely generated subgroup of $\pi_{1}(M)$ must satisfy $\gamma(s) \leq k s^{n}$ for some constant $k$. This is a result of J. Milnor, see [12].

To put this into context, compare with the fact that if $M$ is a compact manifold with negative sectional curvature, $\pi_{1}(M)$ must have exponential growth [12].

The growth results we will describe arise for the most part from volume comparison theorems. Any Riemannian manifold admits a canonical measure, which is determined by the metric. 
Using this measure we can find the volume of the manifold. It is more enlightening, however, to choose a point on the manifold and consider the volume of small disks about that point. Suppose we measure the volume of a (geodesic) disk of radius $R$, then do the same for a disk of radius $R$ in Euclidean space. In general these volumes will be different. For example the disk of radius $\frac{\pi}{2}$ in the standard 2-sphere has volume (area) $=2 \pi$. The corresponding disk in $\mathbb{R}^{2}$ has volume (area) $=\frac{\pi^{3}}{4}$. More generally we have that if all Ricci curvatures of $M^{n}$ are $\geq 0$, for every $x \in M$ :

$$
V(x, R) \leq \omega_{n}(R)
$$

where $V(x, R)$ is the volume of the geodesic ball of radius $R$ about $x$ and $\omega_{n}(R)$ the volume of a ball of radius $R$ in $\mathbb{R}^{n}$. This is the Bishop comparison theorem, see [4; Theorem 3.7]. We also have the Gromov-Bishop comparison theorem [4; Theorem 3.9], which states that

$$
\frac{V(x, R)}{\omega_{n}(R)}
$$

is decreasing with respect to $R$. Thus on manifolds of non-negative Ricci curvature, volume grows slower than in Euclidean space.

We have seen the connection between curvature and volume growth. We will now say a little about the relationship between volume growth and the growth of the fundamental group.

The key fact is that the fundamental group can be regarded as the group of deck transformations of the universal cover. Moreover, if we pull back the metric from the manifold to its universal cover, the deck transformations are actually isometries. Choose a set of generators $\left\{g_{1}, \ldots, g_{r}\right\}$ for $\pi_{1}$ (or if $\pi_{1}$ is not finitely generated consider a finitely generated subgroup of $\pi_{1}$ and select generators for this). Let $M^{n}$ denote our manifold, and let $\tilde{M}$ be its universal cover. Choose a point $x \in \tilde{M}$ and let $D=\max _{i}\left\{d\left(x, g_{i} x\right)\right\}$ where $d(x, y)$ is the distance between $x$ and $y$. Let $N_{R}(x)$ denote the neighbourhood of $\tilde{M}$ centred on $x$ of radius $R$. We can then assert that the neighbourhood $N_{s D}(x),\left(s \in \mathbb{R}^{+}\right)$contains at least $\gamma(s)$ images of $x$ under the action of $\pi_{1}$ (or the subgroup thereof). 
If we consider a disk of volume $V$ about $x$, this disk will be mapped isometrically under the action of $\pi_{1}$ onto at least $\gamma(s)$ other disks which are centred within $N_{s D}(x)$. If this disk is chosen suitably small, we can ensure these images are disjoint. On the other hand, if the disks are suitably large, the images will cover $N_{s D}(x)$. The idea is to estimate the volume of $N_{s D}(x)$ in terms of $\gamma(s) . V$. Invoking a volume comparison theorem then allows us to estimate $\gamma(s)$ in terms of the growth of disks in $\tilde{M}$, and this in turn allows us to estimate the growth of $\gamma(s)$ is terms of $s$.

The first Milnor result above was sharpened by Anderson in [1]. Before discussing his results, let us recall some elementary facts about covering spaces. The universal cover $U$ of a space $S$ is simply-connected and admits a proper discontinuous action from $\pi_{1}(S)$. The quotient of $U$ under this action is $S$. We can also form the quotient of $U$ under the action of a normal subgroup $G \subseteq \pi_{1}(S)$. Such a quotient space will be a (normal) cover of $S$ with deck transformation group isomorphic to $\frac{\pi_{1}(S)}{G}$.

The Milnor results arose from comparing the growth rate of the fundamental group and the growth of volume on the manifold and its universal cover. The Anderson results below are a natural generalization of this. Their importance lies in the fact that they say essentially everything about $\pi_{1}$ growth on non-compact manifolds of non-negative Ricci curvature. They involve comparing the volume growth of a manifold and an arbitrary normal covering space with the growth rate of the group of covering transformations.

Let $M$ be a non-compact manifold with Ric $\geq 0$, and let $N$ be a normal cover of $M$ with covering group $\Gamma$. If $\bar{\Gamma}$ has polynomial growth of order $\geq p$ and $N$ has polynomial volume growth of order $\leq p+k$, then $M$ has polynomial volume growth of order $\leq k([1])$.

This makes intuitive sense for reasons we will discuss. One might expect the curvature of a manifold to be increased by forming the quotient under some group action as this results in a more 'twisted' space. Conversely, one would expect a cover to have smaller curvature and therefore faster volume growth than the original manifold. 
Let $M$ be a manifold of non-negative Ricci curvature which is acted upon properly and discontinuously by two discrete groups $G_{1}$ and $G_{2}$. If the growth of $G_{1}$ is greater than that of $G_{2}$, this suggests that in some sense $G_{1}$ is a larger group than $G_{2}$. We would therefore expect $\frac{M}{G_{1}}$ to be more 'twisted' (ie have greater curvature) than $\frac{M}{G_{2}}$. In terms of volume growth we would expect the volume of small disks to grow slower in $\frac{M}{G_{1}}$ than in $\frac{M}{G_{2}}$. Notice that this is precisely what the above result says: for a given manifold of non-negative Ricci curvature, a lower bound on group growth imposes an upper bound on the volume growth of the quotient space.

Using similar methods of proof it is possible to turn this result around: for a given manifold of non-negative Ricci curvature, an upper bound on the volume growth of a cover imposes an upper bound on the growth of the covering group. More precisely we have: if the volume growth of $M$ is polynomial of order $\geq k$ and $N$ has polynomial volume growth of order $\leq p+k$, then $\Gamma$ has polynomial growth of order $\leq p$.

Using these theorems we can deduce some interesting results about the structure of $\pi_{1}$ in the case that Ric $\geq 0$. Since any finitely generated normal subgroup of $\pi_{1}(M)$ is the group of deck transformations of some covering of $M$, it is easy to see that if the volume growth of our Ricci non-negative manifold is polynomial of order $\geq k$, then every finitely generated subgroup of $\pi_{1}$ has polynomial growth of order $\leq n-k$. Notice that this is generalizes the Milnor theorem.

We can actually say more: a little group theory shows that the first Betti number

$$
b_{1}(M)=\frac{\pi_{1}}{\left[\pi_{1}, \pi_{1}\right]} \leq n-k .
$$

In particular, the maximal rank of a free abelian subgroup of $\pi_{1}$ is $n-k$.

Finally, it is possible to deduce that if the volume growth is maximal, $i e$ of order $n$, then a uniform bound can be imposed on the order of all finitely generated subgroups of $\pi_{1}$, from which it can be concluded that $\pi_{1}$ itself is finite. 
Let us now consider the condition Ric $>0$, again in the noncompact case. First note that the conclusion of Myers' Theorem is false in the non-compact case: there exist manifolds of strictly positive Ricci curvature having infinite fundamental groups. For example Nabonnand [13] showed that a metric of strictly positive Ricci curvature can be given to $S^{1} \times \mathbb{R}^{3}$. What can we say about the growth of $\pi_{1}$ for such manifolds? Clearly the results of Milnor and Anderson for Ricci non-negative manifolds still apply. It is a consequence of [1] that if Ric $>0$ then the order of polynomial growth is $\leq n-3$. As a result of examples of Bérard Bergery [2] and the above result of Nabonnand, it follows that this bound is sharp. The bound does not hold in the Ricci non-negative case: it is easy to construct manifolds for which the fundamental group has order $n-1$ polynomial growth - for example $T^{n-1} \times \mathbb{R}$. However, it is not known to the author whether non-compact manifolds realizing this growth bound exist.

It is natural to ask to what extent our results for Ric $\geq 0$ hold if we perturb this condition slightly, ie allow some small negative curvatures. In other words, are the growth and structure of $\pi_{1}$ stable phenomena?

For compact manifolds the answer is essentially yes. In [16] Wei showed that if $\operatorname{Ric}\left(M^{n}\right) \geq-\epsilon$, then $\pi_{1}(M)$ has polynomial growth of order $\leq n$ provided $\epsilon$ is less than some (small) constant which depends on $n$, the diameter and volume of the manifold.

(There is no stability if we replace Ric by the sectional curvature $K$. Given $\epsilon>0$, any metric with $K$ bounded away from $-\infty$ can be rescaled to satisfy $K \geq-\epsilon$ using some suitably large scaling factor. Ricci curvature, by contrast, is invariant under global rescalings.)

It would be interesting to know if $\pi_{1}$ growth shows similar stability for non-compact manifolds.

We now move to the strongest measure of curvature, the sectional curvature. (The sectional curvature actually determines the curvature tensor -see [4; Proposition 2.1]-and so contains all curvature information.)

If $K>0$ then in the non-compact case, a theorem of Gro- 
moll and Meyer [8] asserts that the manifold must actually be diffeomorphic to $\mathbb{R}^{n}$. From this it follows that the fundamental group is zero. In the compact case there are clearly non-simply connected manifolds with $K>0$, for example the real projective and lens spaces. However, Myers' Theorem ensures that the fundamental group is finite for $K>0$ also. A conjecture due to Chern claims that the fundamental group is actually cyclic. Some progress towards proving this conjecture has been made by Rong [14]. He showed that if the sectional curvature is quarter-pinched, ie $\frac{1}{4}<K \leq 1$ then the fundamental group is almost-cyclic. In other words, there is a cyclic subgroup of finite index.

Weakening positive sectional curvature to non-negative allows much more flexibility for the fundamental group. In the noncompact case, the Soul Theorem [3] describes the structure of manifolds with $K \geq 0$ : all such manifolds are diffeomorphic to the total space of a vector bundle over a compact manifold with $K \geq 0$. From this it follows that the fundamental group must be finitely generated (compare the Milnor problem below). Moreover, a result of Gromov shows that an upper bound for the number of generators needed can be given in terms of the dimension $n$ only (explicitly $2.5^{\frac{n}{2}}$ ). This bound also holds in the compact case. Since non-negative sectional curvature implies non-negative Ricci curvature, the results concerning the growth of the fundamental group of Ricci non-negative manifolds are also true for $K \geq 0$.

No survey of this subject area would be complete without mentioning arguably its most famous unsolved problem. If $M$ is non-compact with non-negative Ricci curvature, then a conjecture of Milnor claims that $\pi_{1}(M)$ itself is finitely generated. Though universally believed to be true, this conjecture has resisted proof for 30 years. If one could establish the truth of this conjecture, it would follow from a result of Gromov [10] that $\pi_{1}(M)$ has a nilpotent subgroup of finite index.

The emphasis in this article has been on consequences of nonnegative curvature. The case of non-positive curvature merits a few words. As mentioned earlier, any manifold - compact or noncompact - of dimension $\geq 3$ admits a metric with negative Ricci 
curvature [11] (and therefore a metric of negative scalar curvature). So admitting a negative or non-positive Ricci curvature has no homotopy-theoretic consequences.

The situation is markedly different for non-positive sectional curvature. The theorem of Hadamard and Cartan [4; Corollary $2.2]$ asserts that the universal cover of such a manifold is $\mathbb{R}^{n}$. As a result, every higher homotopy group vanishes, and all homotopy information is concentrated in the fundamental group. The collection of manifolds which satisfy this curvature requirement is extensive (even in dimension 2 there are infinitely many diffeomorphism types). Not surprisingly, the study of the fundamental group has yielded a correspondingly rich theory. For an introduction to this area, see [5].

The underlying theme of this article has been the interaction of geometry and topology. One would expect the curvature of a manifold to influence a variety of topological invariants-not just the fundamental group. However, with the exception of a few isolated results, very little is known about these relationships. The situation is particularly acute in the case of positive/non-negative Ricci curvature.

\section{References}

[1] M. Anderson, On the topology of complete manifolds of non-negative Ricci curvature, Topology 29 (1990), 41-55.

[2] L. Bérard Bergery, Quelques exemples de variétés Riemanniennes complètes non-compactes à courbure de Ricci positive, C. R. Acad. Sci. Paris, 302 (1986), 159-161.

[3] J. Cheeger and D. Gromoll, On the structure of complete manifolds of non-negative curvature, Ann. of Math. 96 (1972), 413-443.

[4] I. Cheval, Riemannian Geometry. Cambridge University Press: Cambridge, 1993.

[5] P. Eberlein, Global Differential Geometry. MAA Studies in Mathematics vol. 27, 1989.

[6] J. Eschenburg and E. Heintze, An elementary proof of the CheegerGromoll Splitting Theorem, Ann. Glob. Anal. Geom. 2 (1984), 141151. 
[7] D. Gromoll, Spaces of non-negative curvature, Proc. Symp. Pure Math. 54 (1993), 337-356.

[8] D. Gromoll and W. Meyer, On complete open manifolds of positive curvature, Ann. of Math. 90 (1969), 75-90.

[9] M. Gromov, Groups of polynomial growth and expanding maps, Publ. Math. IHES 53 (1981), 53-78.

[10] M. Gromov, Sign and geometric meaning of curvature, IHES 1990.

[11] J. Lohkamp, Metrics of negative Ricci curvature, Ann. of Math. 140 (1994), 655-683.

[12] J. Milnor, A note on curvature and the fundamental group, J. Diff. Geom. 2 (1968), 1-7.

[13] P. Nabonnand, Sur les variétés Riemanniennes complètes à courbure de Ricci positive, C. R. Acad. Sci. Paris 291 (1980), 591-593.

[14] X. Rong, The almost cyclicity of fundamental groups of positively curved manifolds, Invent. Math. 126 (1996), 47-64.

[15] S. Stolz, Simply connected manifolds of positive scalar curvature, Bull. Amer. Math. Soc. 23 (1990), 427-432.

[16] G. Wei, On the fundamental group of manifolds with almost nonnegative Ricci curvature, Proc. Amer. Math. Soc. 110 (1990), 197-199.

David Wraith

Department of Mathematics,

NUI Maynooth,

Co. Kildare,

Ireland. 